\title{
NAMPT: A potential prognostic and therapeutic biomarker in patients with glioblastoma
}

\author{
QIUYUN GUO, NAHAN, LEI SHI, LI YANG, XIAOXI ZHANG, \\ YANGMEI ZHOU, SHIYING YU and MENGXIAN ZHANG \\ Department of Oncology, Tongji Hospital of Tongji Medical College, \\ Huazhong University of Science and Technology, Wuhan, Hubei 430030, P.R. China
}

Received January 8, 2019; Accepted July 3, 2019

DOI: $10.3892 /$ or.2019.7227

\begin{abstract}
Glioblastoma (GBM) is the most common primary intracranial malignancy. GBM still exhibits high recurrence and mortality rates even following combined treatment with surgery, radiotherapy and chemotherapy, Therefore, the identification of novel therapeutic targets is urgent. Previous research has shown that nicotinamide phosphoribosyltransferase (NAMPT) plays a key role in cell metabolism and is closely related to the occurrence and development of many tumor types; yet, little is known concerning its relationship with GBM. Oncomine database analysis showed that the expression of NAMPT in GBM was higher than that in normal tissues; this finding was further confirmed by immunohistochemical staining of a tissue microarray. Data analysis with the R2 platform showed that patients with higher expression of NAMPT had worse prognoses than those with lower NAMPT expression. Using the GBM data in TCGA, four pathways enriched in the high NAMPT expression group were identified by gene set enrichment analysis (GSEA). NAMPT expression was knocked down in U87 and U251 GBM cells by lentiviral vectors carrying a small hairpin RNA (shRNA) targeting NAMPT. CCK-8, colony formation, wound healing, Transwell and apoptosis assays were carried out. The results showed that NAMPT knockdown decreased cell proliferation, migration, and invasion and promoted apoptosis. U87 GBM cells were used in a model of subcutaneous tumorigenesis in nude mice. The results showed that NAMPT knockdown slowed the growth of tumors in vivo. Therefore, we speculate that NAMPT may be a potential prognostic and therapeutic biomarker for glioblastoma.
\end{abstract}

Correspondence to: Dr Mengxian Zhang, Department of Oncology, Tongji Hospital of Tongji Medical College, Huazhong University of Science and Technology, 1095 Jiefang Road, Wuhan, Hubei 430030, P.R. China

E-mail: zhangmx73@163.com

Key words: NAMPT, glioblastoma, shRNA

\section{Introduction}

Glioblastoma is the most common malignant brain cancer diagnosed in humans, with an average patient survival rate of less than one year after diagnosis (1). Surgical resection followed by a combination of radiation and chemotherapy is the standard treatment for glioblastoma. Although innovative therapeutic strategies have continually been investigated in the past two decades, there are still no effective methods to prevent tumor recurrence. Therefore, it is urgent to explore novel chemotherapeutic targets for glioblastoma.

Nicotinamide phosphoribosyltransferase (NAMPT) is a rate-limiting enzyme in the salvage pathway of nicotinamide adenine dinucleotide (NAD) biosynthesis. NAD is an essential coenzyme involved in cellular redox reactions and is a substrate for NAD-dependent enzymes (2). NAD-dependent enzymes, including histone deacetylases (SirT1-T7), CD38 and poly(ADP-ribose) polymerase (PARP), play an important role in the maintenance of organismal metabolic homeostasis and genomic stability (3-5). Thus, NAD displays a wide range of effects on cell cycle progression, apoptosis, DNA repair, circadian rhythms, regulation of chromatin dynamics, telomerase activity, intracellular calcium mobilization, and transcriptional regulation (6-12). All of these effects are associated with the regulation of tumorigenesis and development. NAMPT is a rate-limiting enzyme for NAD synthesis; therefore, it plays an important role in tumor generation and progression (13-15).

Previous studies have shown that NAMPT expression is significantly elevated in various human malignant tumors, including lymphomas, breast cancer, prostate adenocarcinoma, esophageal cancer, gastric cancer, colorectal adenocarcinoma, ovarian serous adenocarcinoma and glioblastoma (6). Higher NAMPT expression is correlated with deeper tumor invasion, presence of lymph node metastases, advanced clinical TMN stage and shorter patient survival times (16-19). To determine the function of NAMPT in glioblastoma, we searched a database and further performed experiments to explore its role in glioblastoma.

\section{Materials and methods}

Oncomine database. The Oncomine database (http://www. oncomine.com) was queried to assess the expression of 
NAMPT. Set filter conditions included: i. Cancer Type: Glioblastoma; ii. Gene: NAMPT; iii. Data Type: mRNA; iv. Sample Type: Clinical Specimen; v. Analysis Type: Cancer vs. Normal Analysis; vi. Critical setting condition: $\mathrm{P}$-value $<1 \times 10^{-4}$, fold change $>2$, gene rank=top $10 \%$.

R2 platform. The Kaplan-Meier survival analysis was performed using the 'Tumor glioblatoma-TCGA-540 MAS5.0-u133a' dataset in the Genomics Analysis and Visualization Platform (R2 platform) (https://hgserver1.amc. $\mathrm{nl} /$ cgi-bin/r2/main.cgi). The median was used as the threshold to distinguish the high and low expression of NAMPT.

Immunohistochemical microarray analysis. A glioblastoma tissue microarray (US Biomax GL805b) was obtained from GeneChem (Shanghai, China) containing two spots each of tissues from 35 cases of glioblastoma, paracarcinoma tissues from 2 cases $(1.5-\mathrm{cm}$ distance from the paracarcinoma tissue to the cancer tissue) and normal neural tissues from 3 cases. A two-step immunohistochemical protocol was used. The primary antibody was an anti-NAMPT rabbit monoclonal antibody (dilution 1:500, cat. no. ab58640; Abcam), and the secondary antibody was from an UltraSensitive SP KIT-9720 (Fujian MXB). Three random high-magnification fields were observed under an optical microscope (Caikon XDS-100, original magnification, $\mathrm{x} 200$ ).

The immunohistochemical microarray staining was scored on a semiquantitative scale by assessing the intensity and proportion of staining. The staining intensity was scored using the following scale: 0 (negative), 1 (weak), 2 (moderate), and 3 (strong). According to the positive rate of staining, the proportion of staining was scored as $0(0-4 \%), 1(5-25 \%)$, $2(26-50 \%), 3(51-75 \%)$, or $4(76-100 \%)$. The total score was calculated by multiplying the proportion score and the intensity score. Specimens with a total score of $\leq 6$ were assigned to the low-expression group. Specimens with a total score of $>6$ were assigned to the high-expression group.

Gene set enrichment analysis (GSEA). GBM gene expression profile was downloaded from The Cancer Genome Atlas (TCGA) database (https://www.cancer.gov/tcga). The queue were divided into NAMPT high and low expression groups according to the median expression of NAMPT. Gene set enrichment analysis was performed using the GSEA software, version 2.0.1, obtained from the Broad Institute (http://www. broad.mit.edu/gsea). Gene set permutations were performed 1,000 times for each analysis. Family error rate (FWER) and false discovery rate (FDR) were used to screen the enriched pathways in each group.

Cell lines and cell culture. The human glioblastoma cell lines, U87 and U251, were obtained from the American Type Culture Collection (ATCC). STR profiling indicated that the U87 was a probable glioblastoma of unknown origin. These cell lines were cultured with Corning ${ }^{\circledR}$ DMEM medium (Corning Inc.) containing $10 \% \mathrm{FBS}$. Cell lines were cultured at $37^{\circ} \mathrm{C}$ in a humidified atmosphere of $5 \% \mathrm{CO}_{2}$.

NAMPT shRNA design and lentivirus construction. A small hairpin RNA (shRNA) targeting NAMPT with high specificity was designed and synthesized by GeneChem (Shanghai, China) and cloned into a lentiviral vector (GeneChem), which also encoded green fluorescent protein (GFP). Then, the lentivirus expressing NAMPT shRNA (LV-NAMPT-RNAi) was prepared and collected. The NAMPT shRNA sequence was as follows: 5'-AACTTAGATGGTCTGGAAT-3'. A scrambled shRNA sequence was used as the negative control: 5'-TTC TCCGAACGTGTCACGT-3'.

Lentivirus infection. The human glioblastoma cell lines U87 and U251 were used for lentivirus infection. Briefly, cells in the logarithmic phase were digested by trypsinase. A $4 \times 10^{4} / \mathrm{ml}$ cell suspension was prepared, and $1 \mathrm{ml}$ of the cell suspension was inoculated into a 12-well plate. When the cell confluence was $20 \%$, the appropriate lentiviral particle solution containing the lentivirus expressing either NAMPT or the scrambled shRNA was added to the target plate (MOI=5). The infection efficiency was determined by the GFP expression status as observed with a fluorescence microscope (Olympus Corp., Tokyo, Japan) $72 \mathrm{~h}$ after infection.

Quantitative real-time PCR ( $q P C R)$. Total RNA was extracted and reverse transcribed. The resulting cDNA was used as the template for RT-PCR using a standard SYBR Green PCR kit (Takara) in a LightCycler 480 Real-Time PCR machine (Roche). Independent experiments were conducted in triplicate. GAPDH served as the internal control. Gene expression was calculated using the $\Delta \Delta \mathrm{Cq}$ method (20).

Western blot analysis. Cell lysates were prepared in ice-cold RIPA lysis buffer. Total protein concentration was determined using an enhanced BCA protein assay kit. An amount of $30 \mu \mathrm{g}$ of total protein per lane was separated by $10 \%$ SDS-PAGE, transblotted onto PVDF membranes, which were then blocked with 5\% milk dissolved in TBST for $1 \mathrm{~h}$ at room temperature and incubated overnight with primary antibodies at $4^{\circ} \mathrm{C}$. The primary antibodies used were as follows: Rabbit anti-NAMPT (cat. no. ab58640; dilution 1:500; Abcam), mouse anti-GAPDH (cat. no. ab8245; dilution 1:3,000; Abcam). Then the blots were incubated with anti-rabbit (cat. no. ab205718; dilution 1:5,000; Abcam) or anti-mouse (cat. no. ab205719; dilution 1:5,000; Abcam) HRP-labeled secondary antibody at room temperature for $1.5 \mathrm{~h}$. The immunoactivity was detected using an ECL-Plus kit (Thermo Fisher Scientific. Inc.). The immunoreactive bands were quantified by densitometry with ImageJ software (National Institutes of Health, Bethesda, MD, USA).

CCK- 8 assay. Cells at a density of 2,000 cells/well in logarithmic phase were cultured in 96-well culture plates. A 10- $\mu$ l volume of CCK-8 reagent (Sigma-Aldrich; Merck KGaA) was added at various time points $(24,48,72,96$ and $120 \mathrm{~h})$. After cells were cultured for $4 \mathrm{~h}$, the absorbance of the plates was read at a wavelength of $450 \mathrm{~nm}$ using a microplate reader (M2009PR; Tecan Infinite). The experiment was repeated five times.

Colony formation assay. Three days after infection with the lentivirus, cells were seeded at a density of 800 cells per well in 6-well plates with triplicate wells for each group. Cell colonies were grown in a humidified $5 \% \mathrm{CO}_{2}$ incubator at $37^{\circ} \mathrm{C}$ for 
16 days. Images were acquired with an inverted microscope at x100 magnification. Then, cells were fixed, stained with Giemsa solution and imaged. Colonies measuring $0.3 \mathrm{~mm}$ or more were counted.

Wound healing assay. Cells at a density of 50,000 cells/well were seeded in 96-well wounding replicator and grown to over $90 \%$ confluence. Then the culture medium was replaced with $0.5 \%$ FBS. Wounds were generated using the tip with $200 \mu \mathrm{m}$ width of a wounding replicator, VP scientific, VP408FH. Cells were incubated for up to $24 \mathrm{~h}$. Wound closure was observed at 0,8 and $24 \mathrm{~h}$ post scratching. These assays were performed with three independent replicates. Cell images were acquired with an inverted microscope (Caikon XDS-100, original magnification, x10). The migration rate was calculated as follows: Migration rate $=[$ (width of wound at $0 \mathrm{~h}$-width of wound at the time point tested)/width of wound at $0 \mathrm{~h}] \times 100 \%$.

Transwell chamber assay. A Corning invasion kit was used to perform this assay according to the manufacturer's instructions. Cell suspensions $\left(10^{5}\right.$ cells per well in a 24 -well culture plate) in FBS-free DMEM were added to the top chamber, and DMEM containing 30\% FBS was added to the bottom chamber. Cells that migrated through the filter were stained with Giemsa. Images of the migrated cells were captured with a digital camera connected to an inverted microscope (Caikon XDS-100). Five random fields of vision at x100 magnification and 9 random fields of vision at $\times 200$ magnification were observed per well. The migrated cells were counted in the $\mathrm{x} 200$ images. The invasion assay was carried out with three independent replicates.

In vivo tumorigenesis in nude mice. The first step was to prepare tumor-forming cells according to the method described above. U87 cells infected with a lentiviral vector expressing NAMPT-shRNA and GFP sequences were generated as the knockdown (KD) group; cells infected with lentiviral vector expressing scrambled shRNA and GFP sequences were generated as the negative control (NC) group. Mice were randomized into the two groups. BALB/c nude mice (male, 4 weeks old, $\sim 20 \mathrm{~g}$ ) were purchased from the HUST Animal Center, Wuhan, China. They were maintained in a constant temperature, humidity, sterile environment and routinely fed with food and water according to the national regulations. A total of $1 \times 10^{7}$ logarithmically growing U87 cells in $0.1 \mathrm{ml}$ of PBS were subcutaneously injected into the right flank of the mice, 10 mice in each group. According to the Institutional Animal Care and Use Committees (IUCUC) protocol (AN-5803), the maximal allowable size of the tumours could not exceed $10 \%$ of mouse body weight. Tumor volumes were measured every four days after injection with a caliper and calculated using the formula $\mathrm{V}=\pi / 6$ (lxwxw), where 1 is the length and $w$ is the width. Forty days after injection, animals were anesthetized using an intraperitoneal injection of $150 \mathrm{mg} / \mathrm{kg}$ pentobarbital sodium. Tumor tissues were excised and weighed. Immunohistochemical staining of Ki-67 and CD31 was performed on the transplanted tumors, and the Ki-67 index and microvascular density (MVD) were calculated. All animal experiments were approved by the
Institutional Animal Care and Use Committee of Huazhong University of Science and Technology.

Statistical analysis. Data are expressed as the means \pm standard errors of the mean. Student's t-test was used to compare the data between two groups. The data between three groups were compared by ANOVA, and then Tukey test was used to compare the data between the two groups if $\mathrm{P}<0.05$. $\mathrm{P}<0.05$ was considered to indicate a statistically significant difference.

\section{Results}

NAMPT is overexpressed in glioblastoma and correlated with poor overall survival. Six studies in the Oncomine database, comprising a total of 942 samples, met the criteria (21-25). Meta-analysis of the 6 studies showed that NAMPT was statistically overexpressed in glioblastoma relative to its expression in normal neural tissue $(\mathrm{P}=1.68 \mathrm{E}-7)$ (Fig. 1A). Immunohistochemical microarray analysis showed that NAMPT was mainly localized in the cytoplasm (Fig. 1C-E). The average total staining scores of the glioblastoma and nontumor tissues (paracarcinoma and normal neural tissues) were 7.54 and 4.600 , respectively $(\mathrm{P}=0.000637)$. These results suggest that the expression of NAMPT in human glioblastoma is higher than that in normal tissues.

Data from the R2 platform (540 glioblastoma samples) showed that patients with higher NAMPT expression had a lower overall survival rate than patients with lower NAMPT expression. The 2-year survival rates were 4 and $1 \%$ for the high- and low-expression groups, respectively $(\mathrm{P}=0.0063)$ (Fig. 1B).

Biological pathways enriched in the NAMPT high-expression phenotype as obtained by gene set enrichment analysis (GSEA). Gene set enrichment analysis (GSEA) was performed using data from the TCGA GBM cohort comprising 169 samples. The expression level of NAMPT was used as the phenotype label. A pathway was classified as enriched by setting the FWER P-value to $<0.01$ and the FDR to $<0.05$. The 'GO acute inflammatory response', 'positive regulation of I- $\kappa \mathrm{B}$ kinase NF- $\kappa$ B signaling', 'I- $\kappa$ B kinase NF- $\kappa$ B signaling', and 'acute phase response' pathways were enriched in the NAMPT high-expression phenotype (Fig. 1F). Therefore, NAMPT may play a role via these pathways.

A lentiviral-based shRNA strategy is successfully used to inhibit NAMPT expression in GBM cells. A lentiviral vector expressing both shRNA targeting human NAMPT and the GFP sequence was used to establish knockdown (KD) cells, while a lentiviral vector expressing both scrambled shRNA and the GFP sequence was used to establish negative control (NC) cells in the U87 and U251 cell lines. Cells without lentivirus infection were considered blank control (CON) cells. GFP expression was observed in more than $90 \%$ of cells infected with the lentivirus $72 \mathrm{~h}$ after infection (Fig. 2A and B).

RT-PCR and western blot analysis were performed to assess the knockdown efficiency. RT-PCR results showed that NAMPT-shRNA was effective in both U251 and U87 GBM cells, with a knockdown efficiency of approximately $74.9 \%$ in U87 GBM cells (Fig. 2C) and 87.7\% in U251 GBM cells 
A Comparison of NAMPT across 6 analyses Over-expression

Median rank p-Value Gene $189.5 \quad 1.68 \mathrm{E}-7$ NAMPT

\section{\begin{tabular}{|l|l|l|l|l|l|}
\hline 1 & 2 & 3 & 4 & 5 & 6 \\
\hline
\end{tabular}}

Legend

$\begin{array}{lc}\text { 1. Glioblastoma vs. Normal } & \text { 4. Glioblastoma vs. Normal } \\ \text { Bredel Brain 2, Cancer Res, 2005 } & \text { Shai Brain, Oncogene, 2003 } \\ \text { 2. Glioblastoma vs. Normal } & \text { 5. Glioblastorna vs. Normal } \\ \begin{array}{l}\text { Lee Brain, Cancer Cell, 2006 } \\ \text { Sun Brain, Cancer Cell, 2006 }\end{array} \\ \begin{array}{l}\text { 3. Glioblastoma vs. Normal } \\ \text { Murat Brain, J Clin Oncol, 2008 }\end{array} & \begin{array}{l}\text { 6. Brain Globlastoma vs. Normal } \\ \text { TCGA Brain, No Associated Paper, 2013 }\end{array}\end{array}$

$\begin{array}{lllll}5 & 1025 \quad 25 & 105 & 1\end{array}$

붐ㅁํㅁำ

The rank for a gene is the median rank for that gene across each of

The p-Value for a gene is its p-Value for the median-fanked analysis.

B

Tumor Glioblastoma-TCGA-540 MAS5.0-u133a

NAMPT (217739_s_at)

Subset: WITH SURV

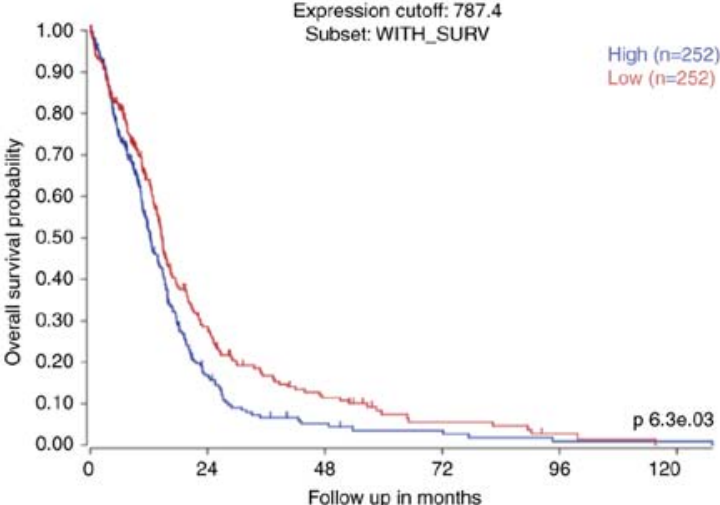

$\mathrm{F}$
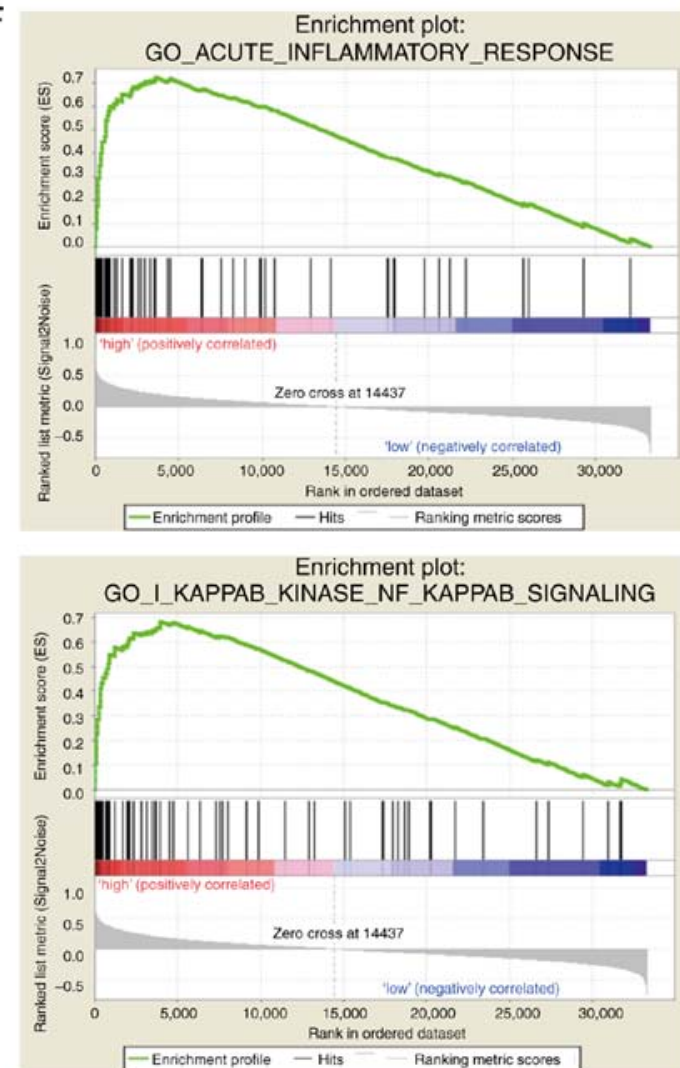

C

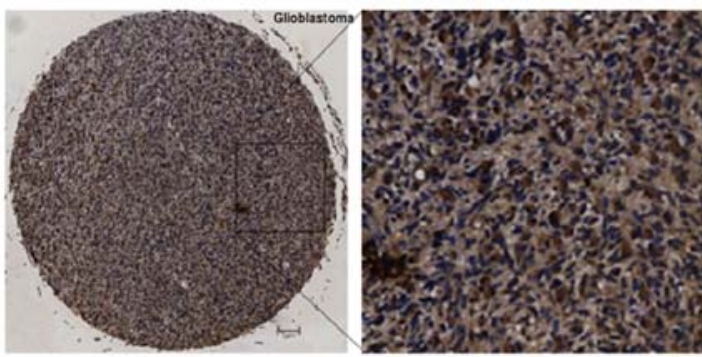

D

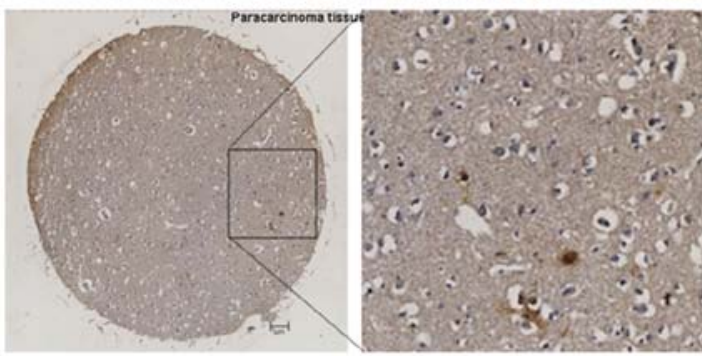

E

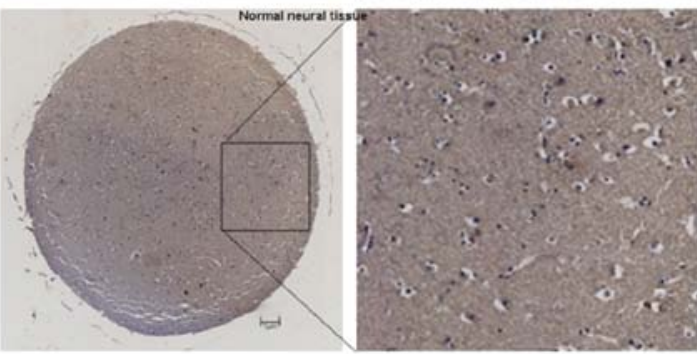

Enrichment plot:GO_ACUTE_PHASE_RESPONSE

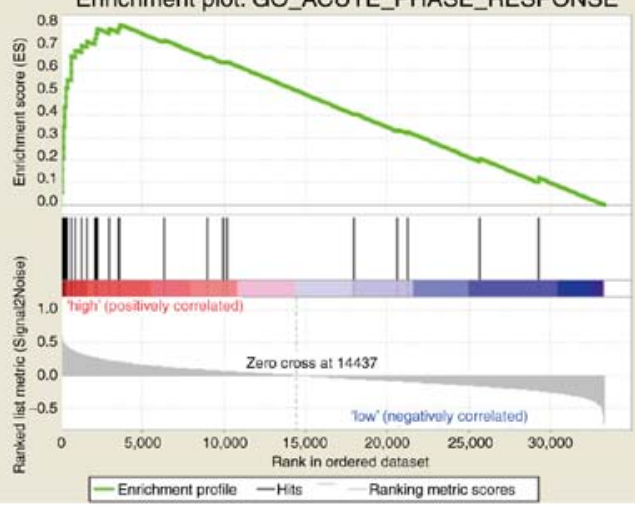

Enrichment plot:

GO_POSITIVE_REGULATION_OF_I_KAPPAB_KINASE_N F_KAPPAB SIGNALING

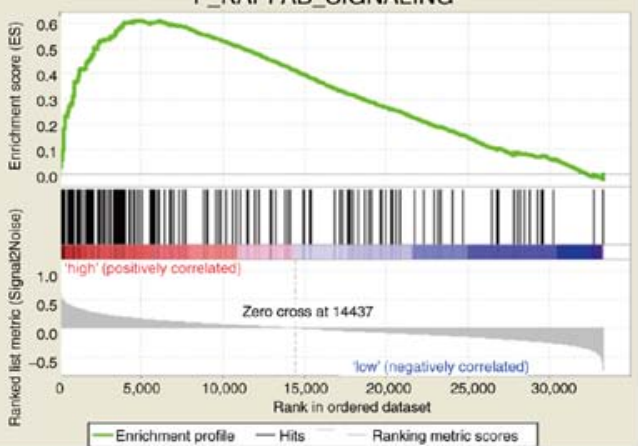

Figure 1. (A) Analysis of the Oncomine database showed that the expression of NAMPT in glioblastoma was higher than that in normal neural tissue. (B) Data analysis with the R2 platform showed that high expression of NAMPT in glioblastoma was associated with a low overall survival rate. (C-E) Immunohistochemical microarray analysis showed that the expression of NAMPT in glioblastoma tissues was higher than that in normal tissues. Representative immunohistochemical images of glioblastoma tissue (C), paracarcinoma tissue (D) and normal neural tissue (E). (F) GSEA analysis using the TCGA database showed that the GO terms 'acute inflammatory response', 'positive regulation of I $\kappa$ B kinase NF- $\kappa B$ signaling', 'I $\kappa$ B kinase NF- $\kappa B$ signaling', and 'acute phase response' were enriched in the NAMPT high-expression phenotype. NAMPT, nicotinamide phosphoribosyltransferase; GO, Gene Ontology; TCGA, The Cancer Genome Atlas. 
A

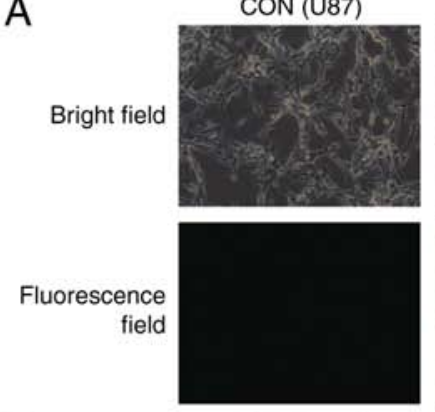

B

Bright field
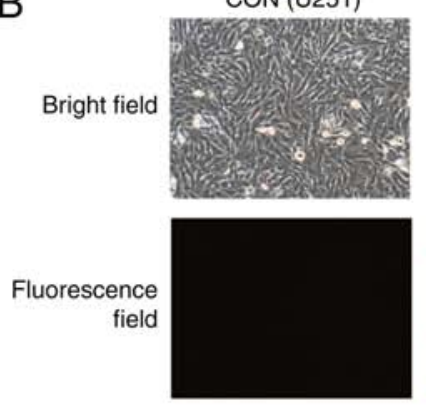

E U87 CON NC KD
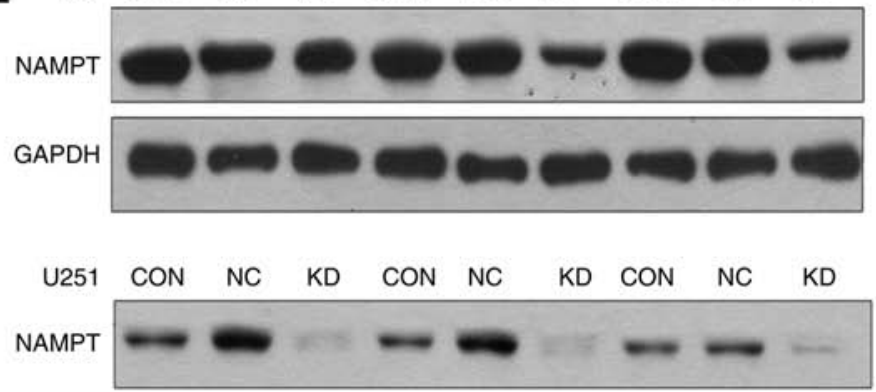

GAPDH

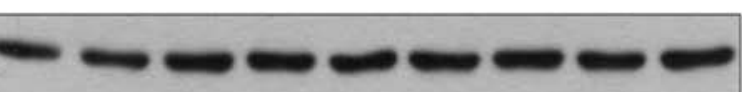

NC (U87)
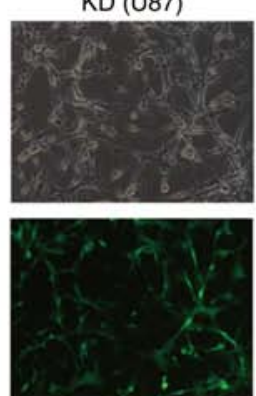

KD (U251)
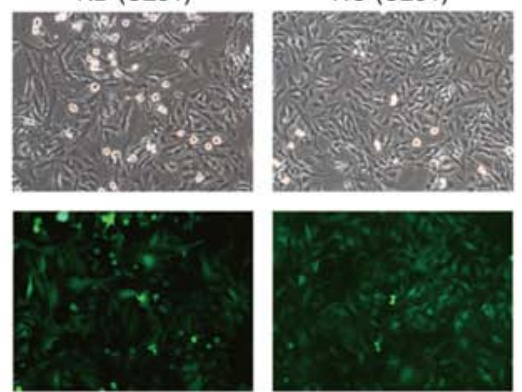

NC (U251)

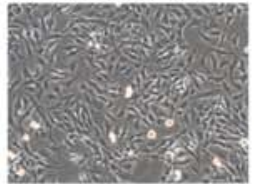

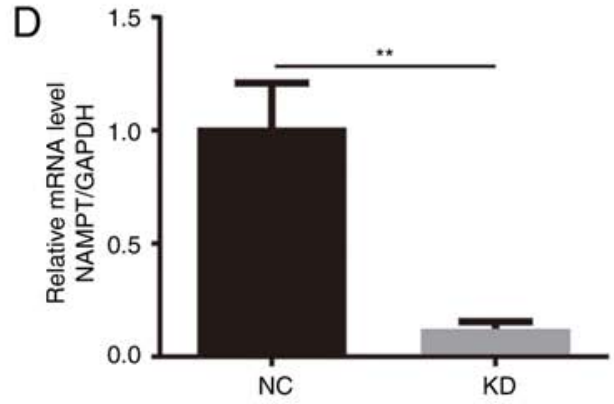

$\mathrm{F}$

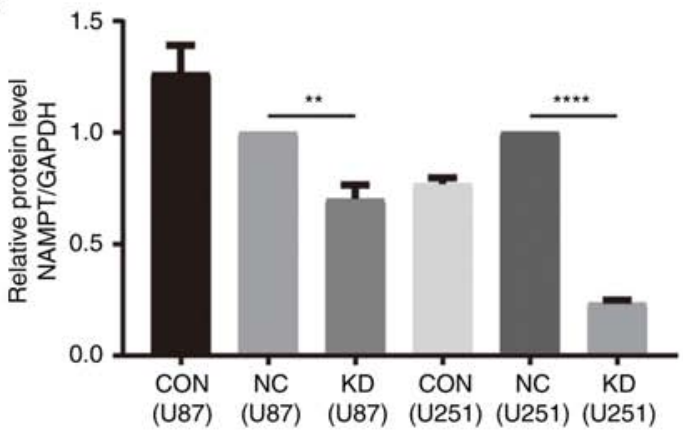

Figure 2. A lentivirus-based shRNA strategy was effectively used to knock down NAMPT in U87 and U251 GBM cell lines. (A and B) Observation of cells via optical and fluorescence microscopy after lentiviral infection (magnification, x200). (C and D) NAMPT-shRNA was effective for NAMPT knockdown in U87 (C) and U251 (D) GBM cells, as verified by RT-PCR. (E and F) NAMPT-shRNA effectively knocked down NAMPT expression in U87 and U251 GBM cells, as verified by western blotting. Western blot band (E) and quantification by Compass software $(\mathrm{F})\left({ }^{*} \mathrm{P}<0.05,{ }^{* *} \mathrm{P}<0.01,{ }^{* * * *} \mathrm{P}<0.0001\right)$. NAMPT, nicotinamide phosphoribosyltransferase; GBM, glioblastoma. Groups: KD, cells transfected with shRNA targeting human NAMPT; NC, cells transfects with scrambled shRNA; CON, cells without lentivirus infection considered as blank control cells.

(Fig. 2D). The location (Fig. 2E) and quantification (Fig. 2F) of the western blot band further confirmed the above results.

NAMPT knockdown inhibits cell proliferation and colony formation in GBM cells. The CCK-8 assay showed that the proliferation of cells in the KD group was significantly inhibited at the 4 and 5 days in U87 and U251 GBM cells $(\mathrm{P}<0.001$ KD vs. NC for both cell lines) (Fig. 3A and B).

The soft agar colony formation assay showed that in the KD, NC and CON groups of U87 and U251 GBM cells the average colony numbers were 19,27 and 40 and 6, 51 and 88 , respectively $(\mathrm{P}<0.05 \mathrm{KD}$ vs. $\mathrm{NC}$ for both cell lines) (Fig. 3C and D).

NAMPT knockdown inhibits cell migration and invasion and induces apoptosis in the GBM cell lines. As shown by the wound healing assay in U87 cells, the migration rates of KD cells at 8 and $24 \mathrm{~h}$ were $0.14 \pm 0.08$ and $0.4 \pm 0.06$, respectively, lower than those of the CON group $(0.21 \pm 0.08$ and $0.64 \pm 0.05)$

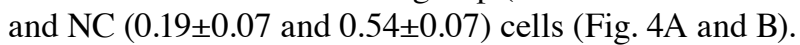

The Transwell assay showed that the average numbers of migrated cells in the $\mathrm{KD}, \mathrm{NC}$ and $\mathrm{CON}$ groups were $56 \pm 5.55$, $101 \pm 3.88$ and $106 \pm 3.74$ in the U87 cells and $16 \pm 0.44,107 \pm 5.00$ and $116 \pm 2.92$ in the $\mathrm{U} 251$ cells, respectively $(\mathrm{P}<0.001$, $\mathrm{KD}$ vs. NC and CON) (Fig. 4C and D).

The apoptosis assay showed that the percentage of apoptotic U87 GBM cells in the KD group was $7.68 \pm 0.3386 \%$, which was higher than that in the NC group $(3.74 \pm 0.3103 \%)(\mathrm{P}<0.001)$. Similarly, the percentage of apoptotic U251 GBM cells in the KD group was $7.43 \pm 0.4921 \%$, which was also higher than that in the NC group $(3.88 \pm 0.3553 \%, \mathrm{P}<0.001)$ (Fig. 4E-G).

NAMPT knockdown inhibits the growth of transplanted U87 GBM cells in nude mice. The growth curve of subcutaneously transplanted tumors in nude mice showed that tumors derived from the KD cells grew much more slowly than 

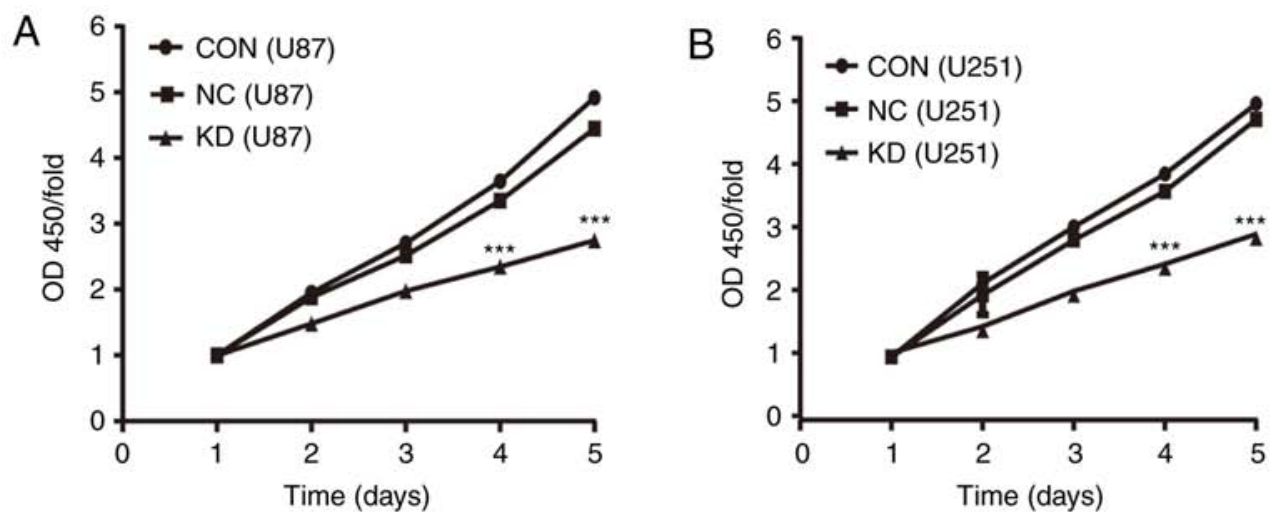

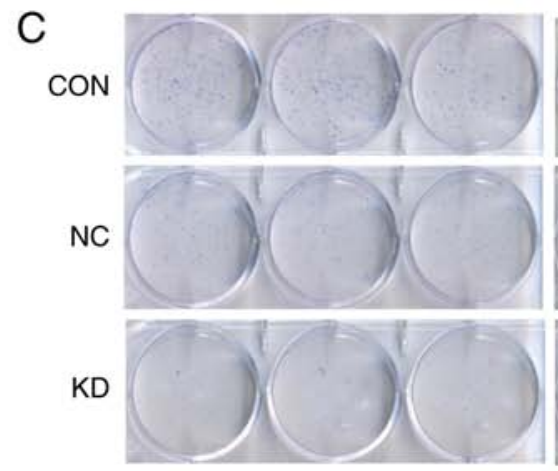

U87

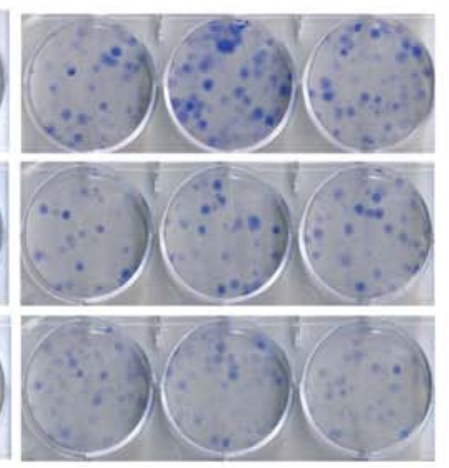

U251

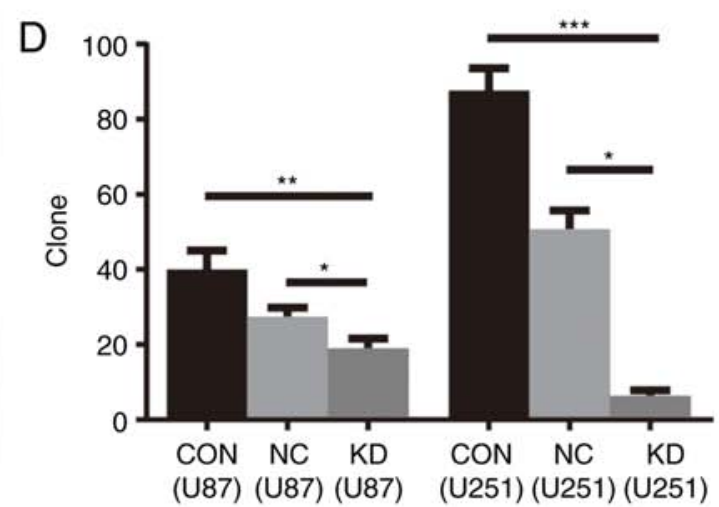

Figure 3. (A and B) Cell proliferation was inhibited by NAMPT knockdown in U87 (A) and U251 (B) GBM cells, as determined by a CCK-8 assay. Cell proliferation data are shown as the comparative fold changes in the OD450 absorbance values on day $1\left({ }^{* * * * *} \mathrm{P}<0.001\right.$ compared with $\left.\mathrm{NC}\right)$. (C and D) Colony formation was decreased by NAMPT knockdown in U87 and U251 GBM cells, as determined by a soft agar assay. Representative images (C) and histogram showing the means \pm SDs of the number of colonies formed in three separate experiments (D) $\left({ }^{*} \mathrm{P}<0.05,{ }^{* *} \mathrm{P}<0.01,{ }^{* * *} \mathrm{P}<0.001\right)$. NAMPT, nicotinamide phosphoribosyltransferase; GBM, glioblastoma. Groups: KD, cells transfected with shRNA targeting human NAMPT; NC, cells transfects with scrambled shRNA; $\mathrm{CON}$, cells without lentivirus infection considered as blank control cells.

those derived from the NC tumors. Forty days after inoculation, the maximum diameter of the KD and $\mathrm{NC}$ transplanted tumors were 11.40 and $18.50 \mathrm{~mm}$, the maximum weights of the KD and NC tumors were 0.643 and $1.049 \mathrm{~g}$, respectively. The average weights of the KD and $\mathrm{NC}$ transplanted tumors were $0.269 \pm 0.244$ and $0.788 \pm 0.186 \mathrm{~g}$, respectively $(\mathrm{P}<0.0001)$ (Fig. 5A-C).

The Ki-67 indices of the KD and NC tumors was 17.7 \pm 3.1 and $55.3 \pm 8.2 \%$, and the MVD in the KD and NC tumors was $15.9 \pm 2.8$ and $28.2 \pm 5.4$, respectively. These results suggest that NAMPT knockdown inhibits the growth and microvessel formation of U87 glioblastoma xenografts in nude mice (Fig. 5D-F).

\section{Discussion}

Manyprevious studies have reported that NAMPT expression is increased in human malignant tumors and that high expression of NAMPT is associated with advanced tumor stage and reduced patient survival time (6,16-19). Hufton et al (26) reported that NAMPT expression was 6-fold higher than that in benign tissues. Olesen et al (18) found that NAMPT was overexpressed in blood diseases and was associated with more aggressive phenotypes of malignant lymphoma. Nakajima et al $(27,28)$ reported that NAMPT may be a useful biomarker for predicting the progression of gastric and colorectal cancer. Maldi et al (16) reported that NAMPT promoted the growth, metastasis and dedifferentiation of melanoma cells. Gujar et al demonstrated that NAMPT is highly expressed in glioblastoma tumors and patient-derived glioblastoma stem-like cells (GSCs), and NAMPT knockdown inhibited the in vivo tumorigenicity of GSCs (29). To further study the effect of NAMPT on glioblastoma, we performed a series of studies. First, we searched the Oncomine database and found that NAMPT was statistically overexpressed in glioblastoma tissues relative to its expression in normal neural tissues. Then, using the R2 platform, we found that high expression of NAMPT was associated with poor prognosis in glioblastoma. Next, we carefully selected a glioblastoma tissue microarray containing tissues from 35 cases (each with two spots). The microarray analysis results indicated that the expression of NAMPT in glioblastoma was higher than that in normal neutral tissue. In conclusion, we believe that NAMPT is overexpressed in glioblastoma and that high NAMPT expression is associated with poor prognosis in glioblastoma.

Inhibition of NAMPT expression by small-molecule inhibitors or gene knockdown has demonstrated antitumor effects both in vitro and in vivo (30-33). Wang et al discovered that inhibition of NAMPT expression in prostate cancer cells suppressed growth and invasion in vitro and the growth of tumor xenografts in vivo (33). Yang et al (34) generated fibrosarcoma HT1080 and 293 cells with stable knockdown of NAMPT using siRNA. The stable cells with knockdown of NAMPT expression were more sensitive to etoposide and had 
A

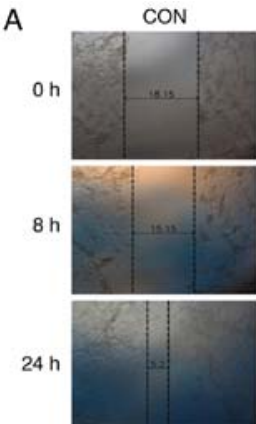

C

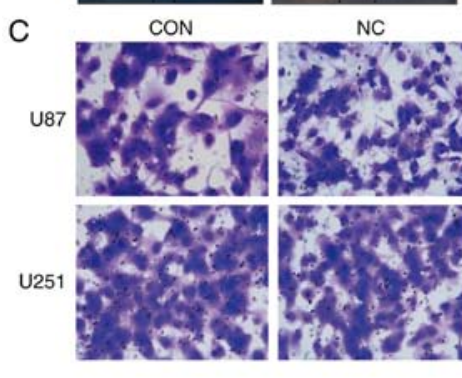

$\mathrm{CON}$
NC

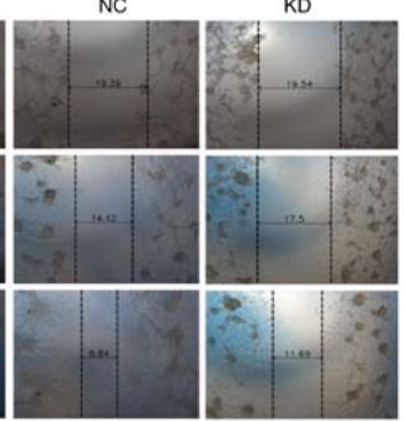

KD

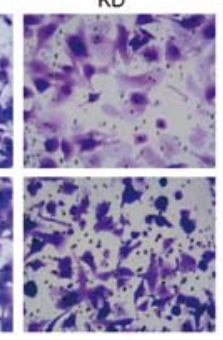

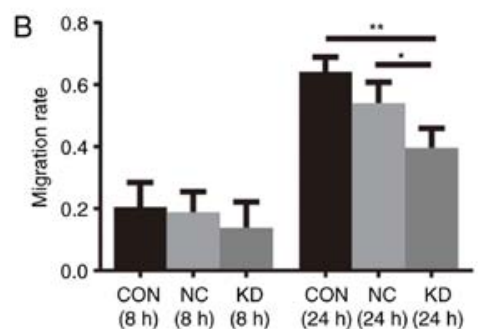

D

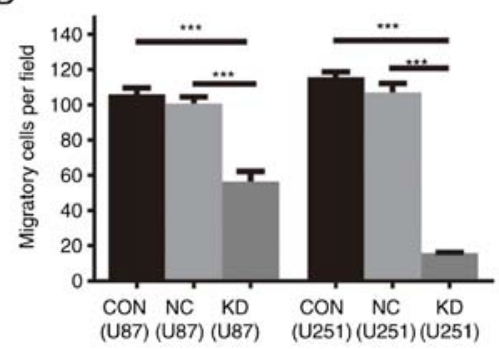

E
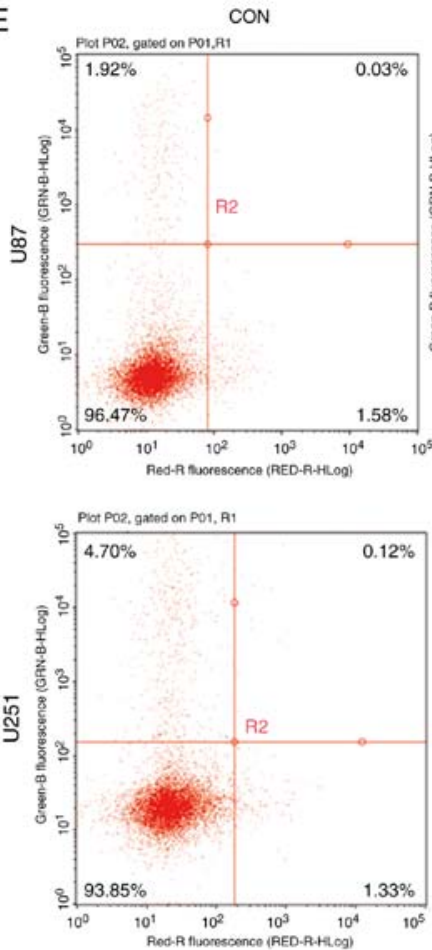

$\mathrm{F}$

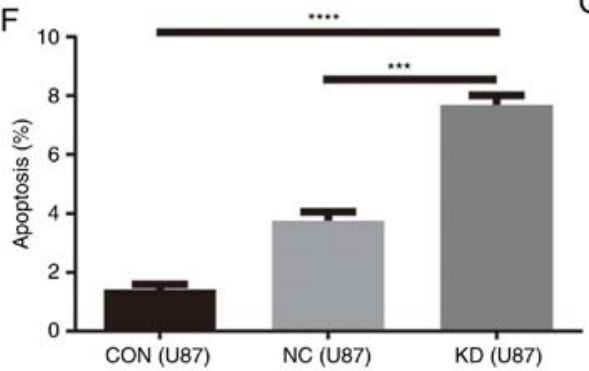

NC
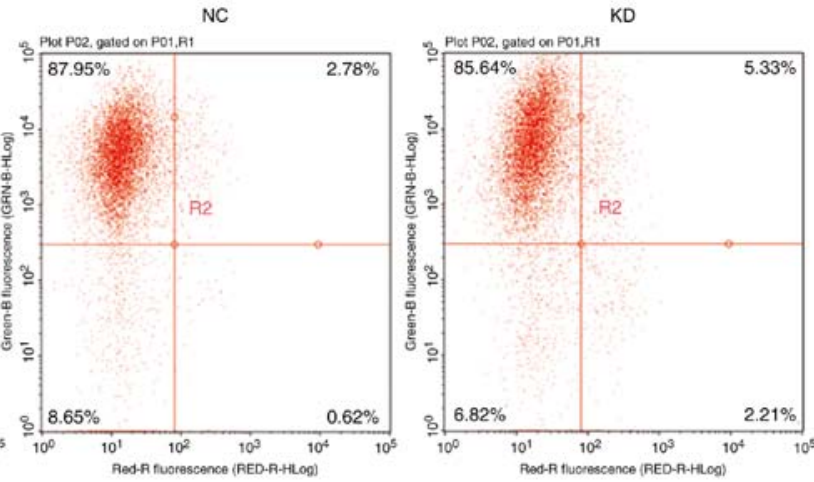

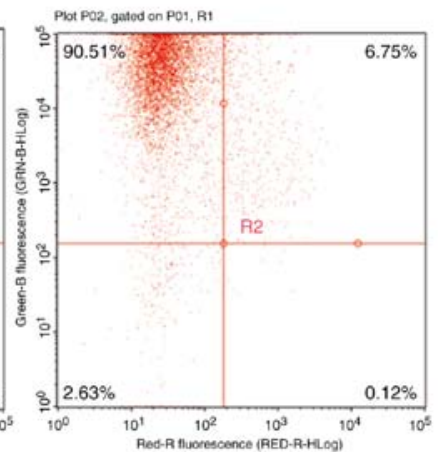

G

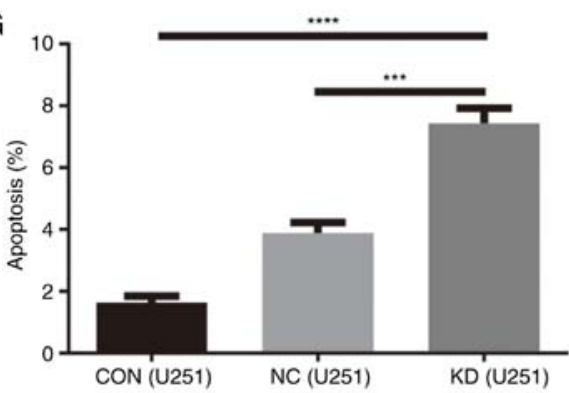

Figure 4. (A and B) Cell migration was decreased by NAMPT knockdown in U87 GBM cells as analyzed by wound healing assay. Representative images (magnification, $\mathrm{x} 100)$. (A) and histogram showing the means \pm SDs of cell migration rate from three separate experiments $\left({ }^{*} \mathrm{P}<0.05,{ }^{* *} \mathrm{P}<0.01\right)$. $(\mathrm{C}$ and $\mathrm{D}) \mathrm{Cell}$ invasion was decreased by NAMPT knockdown in U87 and U251 GBM cells, as determined by a Transwell assay. Representative images (magnification, $\mathrm{x} 200$ ). (C) and histogram showing the means \pm SDs of the number of migrated cells in three separate experiments (D) $\left(^{* * *} \mathrm{P}<0.001\right)$. (E-G) Apoptosis was induced by NAMPT knockdown in U87 and U251 GBM cells. Representative images (E) and histograms showing the means \pm SDs of the percentage of apoptotic cells in three separate experiments in U87 (F) and U251 (G) GBM cells $\left({ }^{* * *} \mathrm{P}<0.001,{ }^{* * * *} \mathrm{P}<0.0001\right)$. NAMPT, nicotinamide phosphoribosyltransferase; GBM, glioblastoma. Groups: KD, cells transfected with shRNA targeting human NAMPT; NC, cells transfects with scrambled shRNA; CON, cells without lentivirus infection considered as blank control cells. 
A
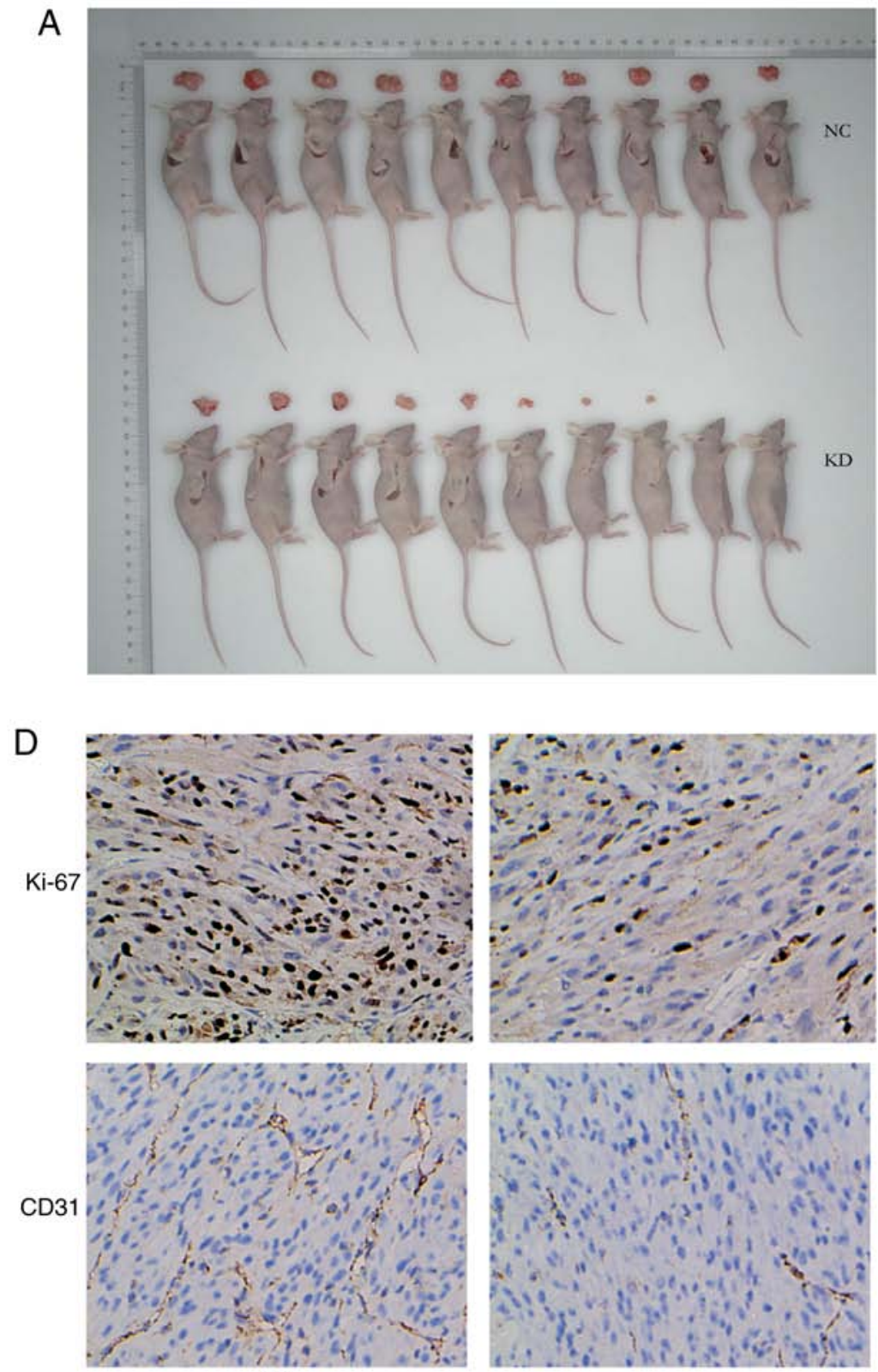

NC
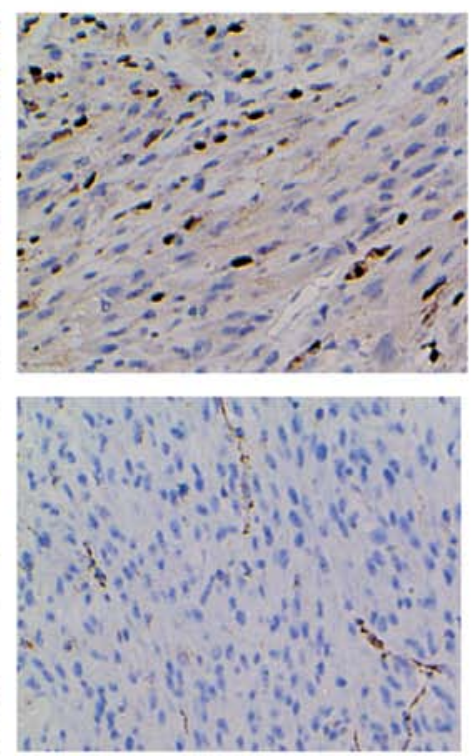

KD

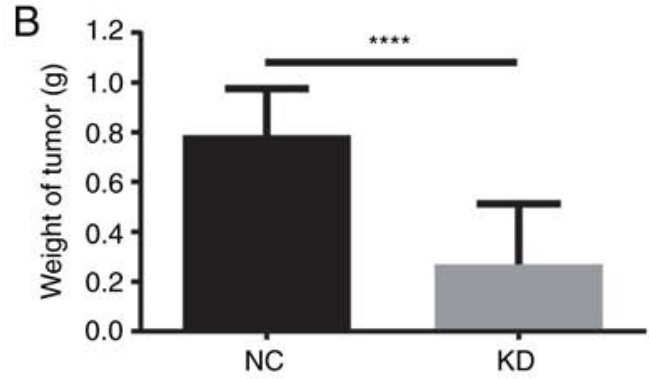

C

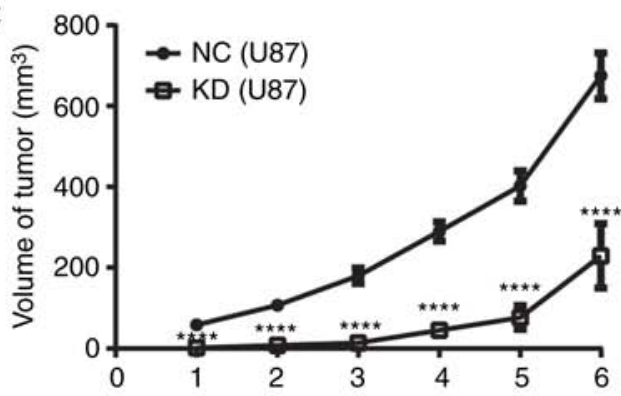

E

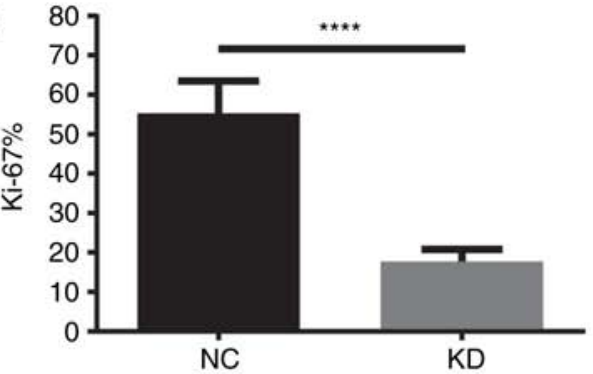

F

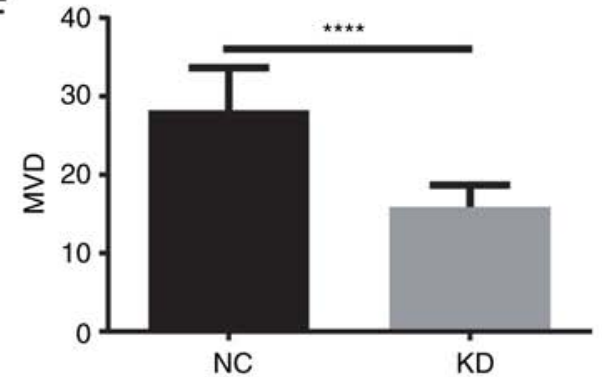

Figure 5. NAMPT knockdown in U87 GBM cells reduces tumor growth in vivo. (A) Images of mice and the corresponding excised xenografts (magnification, $x 100$ ). (B) Histogram showing the mean \pm SD of the tumor weight. (C) Growth curve of tumor volume in nude mice showing that KD tumors grew much more slowly than NC tumors. (D) Representative immunohistochemical images of Ki-67 and CD31 staining. (E) Histogram showing the means \pm SDs of the Ki-67 index. (F) Histogram showing the means \pm SDs of the MVD $\left.{ }^{* * * * *} \mathrm{P}<0.0001\right)$. NAMPT, nicotinamide phosphoribosyltransferase; GBM, glioblastoma. Groups: KD, cells transfected with shRNA targeting human NAMPT; NC, cells transfects with scrambled shRNA; MVD, microvessel density.

increased levels of cleaved caspase 3 than the corresponding parental cells. Jung et al reported that NAD metabolism regulates glioblastoma stem cell maintenance (35). To explore whether NAMPT has an influence on glioblastoma cells, we performed several cytological and animal experiments. We used a lentivirus expressing NAMPT-shRNA to knock down NAMPT expression in two human glioblastoma cell lines, U87 and U251, and confirmed the knockdown effectiveness by RT-PCR and western blotting. Then, we performed CCK- 8 and soft agar assays and observed that cell proliferation was inhibited by NAMPT knockdown. In addition, apoptosis analysis showed that NAMPT knockdown induced apoptosis. The wound healing and Transwell assays showed that cell migration and invasion were decreased by NAMPT knockdown. Our data confirmed that NAMPT knockdown markedly inhibited cell proliferation, migration and invasion and induced apoptosis in U87 and U251 GBM cell lines. To further confirm our conclusion in vivo, we established a subcutaneous xenograft model in nude mice. The growth of xenografts was significantly slowed by NAMPT knockdown. Immunohistochemical staining of xenografts showed that the Ki-67 index and microvessel density (MVD) of NAMPT knockdown tumors were lower than those of negative control tumors. These results showed that NAMPT knockdown exerts a significant inhibitory effect on the tumorigenesis of GBM cells in vitro and in vivo. 
The exact molecular mechanism by which NAMPT regulates glioblastoma generation and progression is complicated and remains unclear. Studies have shown that NAMPT may perform its function through NAD, which is an essential cofactor involved in cellular redox reactions and a substrate for NAD-dependent enzymes such as poly(ADP-ribose) polymerases (PARPs), sirtuins, and cyclic ADP (cADP) ribose synthases. PARP-1 is an important regulator of cell stress and responses to DNA damage. Venkateshaiah et al found that NAMPT inhibition lowers cellular PARP-1 activity and cell viability in myeloma cells (36). Sirtuin1 can attenuate p53, PTEN, and retinoblastoma protein activity; stabilize N-Myc; promote epithelial-to-mesenchymal transition; and increase cell migration (17). Revollo et al found that increased NAMPT expression increases cellular NAD levels and enhanced Sirtuin1-mediated transcription in murine cells (37). Our GSEA results indicated that the GO terms 'acute inflammatory response', 'positive regulation of $\mathrm{I} \kappa \mathrm{B}$ kinase $\mathrm{NF}-\kappa \mathrm{B}$ signaling', 'I $\kappa \mathrm{B}$ kinase $\mathrm{NF}-\kappa \mathrm{B}$ signaling', and 'acute phase response' were enriched in the NAMPT high-expression phenotype. Therefore, we deduced that NAMPT may play its role through these pathways. However, we have not experimentally verified the specific role and mechanism of NAMPT in these pathways, which may be one of our next research directions.

In summary, our study demonstrated that NAMPT is significantly overexpressed in glioblastoma tissues and that high NAMPT expression indicates worse prognosis. In vitro and in vivo experiments showed that NAMPT knockdown reduced cell proliferation, migration, and invasion and induced apoptosis. Therefore, NAMPT may be considered an oncogene in glioblastoma and may be a potential prognostic and therapeutic target for glioblastoma. In the future, we may further study the antitumor mechanism of NAMPT through four pathways derived from the GSEA analysis, and ascertain whether NAMPT knockdown or NAMPT inhibitors combined with chemotherapy, radiotherapy or targeted drugs can play an unexpected antitumor role in glioblastoma.

\section{Acknowledgements}

Not applicable.

\section{Funding}

This study was funded by the National Natural Science Foundation (grant no. 1772680).

\section{Availability of data and materials}

The datasets used and/or analyzed during the current study are available from the corresponding author on reasonable request.

\section{Authors' contributions}

QG performed the cell biology and animal experiments and wrote the manuscript. MZ conducted the cell biology and animal experiments, and managed the project. $\mathrm{NH}$ performed the bioinformatic data analysis and contributed to the project management. LS contributed to the management of the project and performed the cell biology experiments. LY contributed to the animal experiments and project management. $\mathrm{XZ}$ helped conduct the cell biology experiments and modify the manuscript. YZ contributed to the cell biology experiments and modified the manuscript. SY contributed to the animal experiments and managed the project. All authors read and approved the final manuscript and agreed to be accountable for all aspects of the work in ensuring that questions related to the accuracy or integrity of any part of the work are appropriately investigated and resolved.

\section{Ethics approval and consent to participate}

The animal experiments in the present study were approved by the Ethics Committee for Animal Experimentation of Genechem (no. GSZE0116844).

\section{Patient consent for publication}

Not applicable.

\section{Competing interests}

The authors declare that they have no competing interests.

\section{References}

1. Iacob G and Dinca EB: Current data and strategy in glioblastoma multiforme. J Med Life 2: 386-393, 2009.

2. Garten A, Schuster S, Penke M, Gorski T, de Giorgis T and Kiess W: Physiological and pathophysiological roles of NAMPT and NAD metabolism. Nat Rev Endocrinol 11: 535-546, 2015.

3. Imai S, Armstrong CM, Kaeberlein M and Guarente L: Transcriptional silencing and longevity protein Sir2 is an NAD-dependent histone deacetylase. Nature 403: 795-800, 2000.

4. Kim MY, Mauro S, Gevry N, Lis JT and Kraus WL: $\mathrm{NAD}^{+}$-dependent modulation of chromatin structure and transcription by nucleosome binding properties of PARP-1. Cell 119: 803-814, 2004

5. Van Gool F, Galli M, Gueydan C, Kruys V, Prevot PP, Bedalov A, Mostoslavsky R, Alt FW, De Smedt T and Leo O: Intracellular NAD levels regulate tumor necrosis factor protein synthesis in a sirtuin-dependent manner. Nat Med 15: 206-210, 2009.

6. Shackelford RE, Mayhall K, Maxwell NM, Kandil E and Coppola D: Nicotinamide phosphoribosyltransferase in malignancy: A review. Genes Cancer 4: 447-456, 2013.

7. Lin SJ and Guarente L: Nicotinamide adenine dinucleotide, a metabolic regulator of transcription, longevity and disease. Curr Opin Cell Biol 15: 241-246, 2003.

8. Sebastian C, Satterstrom FK, Haigis MC and Mostoslavsky R: From sirtuin biology to human diseases: An update. J Biol Chem 287: 42444-42452, 2012.

9. Chiarugi A, Dolle C, Felici R and Ziegler M: The NAD metabolome-a key determinant of cancer cell biology. Nat Rev Cancer 12: 741-752, 2012.

10. Chinnadurai G: The transcriptional corepressor CtBP: A foe of multiple tumor suppressors. Cancer Res 69: 731-734, 2009.

11. Malavasi F, Deaglio S, Funaro A, Ferrero E, Horenstein AL, Ortolan E, Vaisitti T and Aydin S: Evolution and function of the ADP ribosyl cyclase/CD38 gene family in physiology and pathology. Physiol Rev 88: 841-886, 2008.

12. Burkle A and Virag L: Poly(ADP-ribose): PARadigms and PARadoxes. Mol Aspects Med 34: 1046-1065, 2013.

13. Rodgers JT, Lerin C, Gerhart-Hines Z and Puigserver P: Metabolic adaptations through the PGC-1 alpha and SIRT1 pathways. FEBS Lett 582: 46-53, 2008.

14. van der Horst A, Tertoolen LG, de Vries-Smits LM, Frye RA, Medema RH and Burgering BM: FOXO4 is acetylated upon peroxide stress and deacetylated by the longevity protein hSir2(SIRT1). J Biol Chem 279: 28873-28879, 2004. 
15. Bordone L, Motta MC, Picard F, Robinson A, Jhala US, Apfeld J, McDonagh T, Lemieux M, McBurney M, Szilvasi A, et al: Sirt1 regulates insulin secretion by repressing $\mathrm{UCP} 2$ in pancreatic beta cells. PLoS Biol 4: e31, 2006.

16. Maldi E, Travelli C, Caldarelli A, Agazzone N, Cintura S, Galli U, Scatolini M, Ostano P, Miglino B, Chiorino G, et al: Nicotinamide phosphoribosyltransferase (NAMPT) is over-expressed in melanoma lesions. Pigment Cell Melanoma Res 26: 144-146, 2013.

17. Huang WS, Chen CN, Sze CI and Teng CC: Visfatin induces stromal cell-derived factor- 1 expression by $\beta 1$ integrin signaling in colorectal cancer cells. J Cell Physiol 228: 1017-1024, 2013.

18. Olesen UH, Hastrup N and Sehested M: Expression patterns of nicotinamide phosphoribosyltransferase and nicotinic acid phosphoribosyltransferase in human malignant lymphomas. APMIS 119: 296-303, 2011.

19. Reddy PS, Umesh S, Thota B, Tandon A, Pandey P, Hegde AS, Balasubramaniam A, Chandramouli BA, Santosh V, Rao MR, et al: PBEF1/NAmPRTase/Visfatin: A potential malignant astrocytoma/glioblastoma serum marker with prognostic value. Cancer Biol Ther 7: 663-668, 2008.

20. Livak KJ and Schmittgen TD: Analysis of relative gene expression data using real-time quantitative PCR and the 2(-Delta Delta C(T)) method. Methods 25: 402-408, 2001

21. Shai R, Shi T, Kremen TJ, Horvath S, Liau LM, Cloughesy TF, Mischel PS and Nelson SF: Gene expression profiling identifies molecular subtypes of gliomas. Oncogene 22: 4918-4923, 2003.

22. Bredel M, Bredel C, Juric D, Harsh GR, Vogel H, Recht LD and Sikic BI: Functional network analysis reveals extended gliomagenesis pathway maps and three novel MYC-interacting genes in human gliomas. Cancer Res 65: 8679-8689, 2005.

23. Lee J, Kotliarova S, Kotliarov Y, Li A, Su Q, Donin NM, Pastorino S, Purow BW, Christopher N, Zhang W, et al: Tumor stem cells derived from glioblastomas cultured in bFGF and EGF more closely mirror the phenotype and genotype of primary tumors than do serum-cultured cell lines. Cancer Cell 9: 391-403, 2006.

24. Sun L, Hui AM, Su Q, Vortmeyer A, Kotliarov Y, Pastorino S, Passaniti A, Menon J, Walling J, Bailey R, et al: Neuronal and glioma-derived stem cell factor induces angiogenesis within the brain. Cancer Cell 9: 287-300, 2006

25. Murat A,MigliavaccaE,Gorlia T,Lambiv WL,ShayT,HamouMF, de Tribolet N, Regli L, Wick W, Kouwenhoven MC, et al: Stem cell-related 'self-renewal' signature and high epidermal growth factor receptor expression associated with resistance to concomitant chemoradiotherapy in glioblastoma. J Clin Oncol 26: 3015-3024, 2008.

26. Hufton SE, Moerkerk PT, Brandwijk R, de Bruine AP, Arends JW and Hoogenboom HR: A profile of differentially expressed genes in primary colorectal cancer using suppression subtractive hybridization. Febs Lett 463: 77-82, 1999.
27. Nakajima TE, Yamada Y, Hamano T, Furuta K, Gotoda T, Katai H, Kato K, Hamaguchi T and Shimada Y: Adipocytokine levels in gastric cancer patients: Resistin and visfatin as biomarkers of gastric cancer. J Gastroenterol 44: 685-690, 2009.

28. Nakajima TE, Yamada Y, Hamano T, Furuta K, Matsuda T, Fujita S, Kato K, Hamaguchi T and Shimada Y: Adipocytokines as new promising markers of colorectal tumors: Adiponectin for colorectal adenoma, and resistin and visfatin for colorectal cancer. Cancer Sci 101: 1286-1291, 2010.

29. Gujar AD, Le S, Mao DD, Dadey DY, Turski A, Sasaki Y, Aum D, Luo J, Dahiya S, Yuan L, et al: An NAD ${ }^{+}$-dependent transcriptional program governs self-renewal and radiation resistance in glioblastoma. Proc Natl Acad Sci USA 113: E8247-E8256, 2016.

30. Lee YC, Yang YH, Su JH, Chang HL, Hou MF and Yuan SS: High visfatin expression in breast cancer tissue is associated with poor survival. Cancer Epidemiol Biomarkers Prev 20: 1892-1901, 2011.

31. Bi TQ, Che XM, Liao XH, Zhang DJ, Long HL, Li HJ and Zhao W: Overexpression of Nampt in gastric cancer and chemopotentiating effects of the Nampt inhibitor FK866 in combination with fluorouracil. Oncol Rep 26: 1251-1257, 2011.

32. Shackelford RE, Bui MM, Coppola D and Hakam A: Over-expression of nicotinamide phosphoribosyltransferase in ovarian cancers. Int J Clin Exp Pathol 3: 522-527, 2010.

33. Wang B, Hasan MK, Alvarado E, Yuan H, Wu H and Chen WY: NAMPT overexpression in prostate cancer and its contribution to tumor cell survival and stress response. Oncogene 30: 907-921, 2011.

34. Yang H, Yang T, Baur JA, Perez E, Matsui T, Carmona JJ, Lamming DW, Souza-Pinto NC, Bohr VA, Rosenzweig A, et al: Nutrient-sensitive mitochondrial $\mathrm{NAD}^{+}$levels dictate cell survival. Cell 130: 1095-1107, 2007.

35. Jung J, Kim LJ, Wang X, Wu Q, Sanvoranart T, Hubert CG, Prager BC, Wallace LC, Jin X, Mack SC and Rich JN: Nicotinamide metabolism regulates glioblastoma stem cell maintenance. JCI insight 2: pii: 90019, 2017.

36. Venkateshaiah SU, Khan S, Ling W, Bam R, Li X, van Rhee F, Usmani S, Barlogie B, Epstein J and Yaccoby S: NAMPT/PBEF1 enzymatic activity is indispensable for myeloma cell growth and osteoclast activity. Exp Hematol 41: 547-557.e2, 2013.

37. Revollo JR, Grimm AA and Imai S: The regulation of nicotinamide adenine dinucleotide biosynthesis by Nampt/PBEF/visfatin in mammals. Curr Opin Gastroenterol 23: 164-170, 2007.

This work is licensed under a Creative Commons Attribution-NonCommercial-NoDerivatives 4.0 International (CC BY-NC-ND 4.0) License. 